\title{
COMMUNICATION
}

\section{L'ostéo-odonto-kératoprothèse : la dent et son parodonte au secours du globe oculaire.}

\section{Urban $\mathbf{M}^{1}$, Muraine $\mathbf{M}^{2}$}

1. Service d'Odontologie, Hôpital Charles Nicolle - CHU Rouen

2. Service d'Ophtalmologie, Hôpital Charles Nicolle - CHU Rouen

\section{Introduction}

L'ostéo-odonto-kératoprothèse (OOKP) est une technique chirurgicale mise au point en 1963 en Italie par le Docteur Benedetto Strampelli [1], puis reprise et modifiée par le Docteur Giancarlo Falcinelli. Le principe consiste au remplacement prothétique de la cornée (appelé kératoprothèse) chez des patients souffrant d'une cécité d'origine cornéenne et qui ne peuvent être éligibles à une greffe de cornée classique (syndrome sec sévère, brûlures chimiques ou thermiques).

\section{Observation}

La dent et son parodonte [2] (périoste - os alvéolaire - ligament alvéolo-dentaire) sont prélevés afin de servir de support biologique appelé « lame ostéo-dentaire » à une optique de synthèse de polymétacrylate de méthyl, constituant ainsi I'OOKP, suturée à la cornée, dont les dimensions finales sont de l'ordre de $8 \mathrm{~mm}$ de large par 14-16 mm de long par 2-4 $\mathrm{mm}$ d'épaisseur. Cette OOKP, une fois suturée à la cornée, est recouverte d'un lambeau de muqueuse jugale assurant une couverture biologique du dispositif. L'OOKP joue ainsi de rôle de la cornée permettant de conduire l'influx lumineux à la rétine.

Le tissu dentaire et son parodonte sont privilégiés par rapport aux autres tissus durs de l'organisme car ils présentent notamment des propriétés de stabilité sur le long terme indispensables à la survie de la kératoprothèse.

La dent sélectionnée est le plus souvent une canine[3], car c'est souvent elle qui présente la racine la plus longue et la plus large, nécessaire à l'élaboration de l'OOKP . Cette dent doit être saine, sans traitement canalaire, sans restauration coronaire ni récession gingivale.

Cette technique chirurgicale se divise en deux temps opératoires généralement espacés de 2 à 3 mois. Le temps chirurgical oral est capital pour le succès de l'OOKP qui dépend de la qualité de prélèvement de la dent candidate et de son tissu de soutien. Le chirurgien oral intervient au temps muqueux (prélèvement de la muqueuse jugale), au temps buccal (prélèvement du complexe ostéo-dentaire et reconstruction du site), mais aussi lors du remodelage du complexe ostéo-dentaire en OOKP.

\section{Discussion}

Il apparaît impératif, à la vue de la taille du défaut osseux généré, de s'interroger sur les possibilités de reconstruction et de réhabilitation de la sphère bucco-dentaire. Actuellement, la gestion du défaut consiste en un comblement peropératoire à l'aide de biomatériaux. Les solutions de réhabilitation pouvant être envisagées sont fonction du défaut osseux résiduel, de la taille et du type de l'édentement, mais aussi des attentes esthétiques et possibilités financières du patient. Ce peut être des implants, de la prothèse amovible ou fixée.

\section{Conclusion}

L'OOKP constitue le gold standard en présence d'un syndrome sec sévère en permettant une réhabilitation visuelle de qualité et stable dans le temps. Néanmoins c'est une procédure lourde, irréversible et chronophage qui nécessite une collaboration totale entre l'équipe de chirurgie orale et d'ophtalmologistes.

marion.urban@orange.fr

\section{Références}

1.Meenu J, Vineet V et al. Modified osteo-odonto-keratoprosthesis. Journal of Dental Science \& Oral Rehabilitation. Oct-Dec 2011:18-19.

2.Narayanan V, Nirvikalpa N et al. Osteo-odonto-keratoprosthesis - a maxillofacial perspective. J Craniomaxillofac Surg. 2012 Dec;40(8):e426-31. 3.Sawatari Y, L. Perez V et al. Oral and maxillofacial surgeons' role in the first successful modified osteo-odonto-keratoprosthesis performed in the United States. J Oral Maxillofac Surg. 2011 Jun;69(6):1750-6.

(C) The authors, published by EDP Sciences. This is an Open Access article distributed under the terms of the Creative Commons Attribution License 4.0 (http://creativecommons.org/licenses/by/4.0/). 